\title{
The Power Smith Tube: Joint Optimization of Power Amplifier Input Power and Load Impedance for Power-Added Efficiency and Adjacent- Channel Power Ratio
}

\author{
Joseph Barkate, Matthew Fellows, Jennifer Barlow, Charles Baylis, and Robert J. Marks II \\ Wireless and Microwave Circuits and Systems Program \\ Department of Electrical and Computer Engineering \\ Baylor University \\ Waco, TX, USA
}

\begin{abstract}
A new visualization tool for design is introduced: the Power Smith Tube. It provides aid in designing for optimum power-added efficiency (PAE) while meeting adjacent-channel power ratio (ACPR) requirements. The Power Smith Tube is a three-dimensional cylindrical extension of the Smith Chart with input power as the vertical axis. As such, this Smith Tube allows the visualization of design metrics, such as PAE and ACPR, as a function of both input power and load impedance. Performance of load-pull measurements or simulations at multiple values of input power provides data for the design. Design examples using the Power Smith Tube based on both simulation and measurement data are presented to demonstrate selection of load impedance and input power providing the highest PAE under ACPR constraints.
\end{abstract}

Keywords - power-added efficiency, adjacent-channel power ratio, load-pull, power amplifiers, Smith Chart, optimization

\section{INTRODUCTION}

The design of power amplifiers requires the inclusion of many factors into the design process. Both linearity and efficiency depend significantly on both the load reflection coefficient $\Gamma_{L}$ and the input power $P_{i n}$. Typically, a value of $\Gamma_{L}$ providing desirable power-added efficiency (PAE) and adjacent-channel power ratio (ACPR) characteristics might be selected from load-pull measurements at a selected $P_{i n}$, and then a power sweep might be performed (or vice versa) to hone in on the best value of $P_{i n}$. The impedance is then tuned again. This process is often iterated multiple times, and can frustrate designers because of the need to bounce "back and forth" from load-pull measurements to power sweep data.

This paper introduces the Power Smith Tube to provide visualization of PAE and ACPR variation over all combinations of $\Gamma_{L}$ and $P_{i n}$. This visual aid will enhance convergence to design solutions and assist in creating optimization algorithms for simulation-based design. The Power Smith Tube is also expected to be a convenient optimization space for real-time, joint optimizations of input power and reflection coefficient in reconfigurable power amplifiers.

The Power Smith Tube is shown in Fig. 1: a cylindrical extension of the Smith Chart with input power as the third dimension. It is similar to the Smith Tube presented by Fellows [1], but the vertical axis represents input power instead of waveform bandwidth.

Designing for efficiency and linearity performance over varying input power can be challenging, for example, with high peak-to-average power ratio signals in wireless communications. Snider [2] and Nemati [3] show that the optimum-efficiency load can vary with input power. $\mathrm{Fu}$ demonstrates a varactor-based tunable matching network to improve both efficiency and linearity [4]. Hajji analyzes the necessary change in input power for constant intermodulation rejection [5]. The Power Smith Tube is a unique extension of the Smith Chart to incorporate input power into the design. Previous extensions of the Smith Chart incorporate negative impedances [6, 7], fractional circuit elements [8], lossy transmission lines [9], and waveform bandwidth [1].

The Power Smith Tube can be used to find the combination of $\Gamma_{L}$ and $P_{\text {in }}$ producing the largest PAE while remaining within the ACPR constraint. From load-pull measurements performed at different values of $P_{i n}$, a surface of all points with the limiting value of ACPR can be plotted in the Smith Tube. The point with the largest PAE that meets ACPR requirements is identified as the constrained optimum.

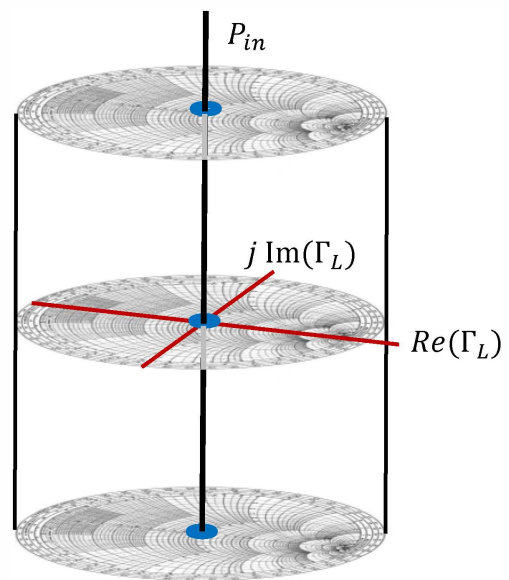

Fig. 1. The Power Smith Tube. The vertical axis represents input power, and the horizontal cross section is a conventional Smith chart. 
Typical power compression curves show that, for increasing $P_{i n}$, PAE increases to a maximum and then drops off again at very high power. This trend generates an expectation that the region of the Smith Chart containing a given PAE or higher (cross-section of a Smith Tube surface of constant PAE) is expected to widen as the Smith Tube is ascended, and then narrow again following the optimum $P_{\text {in }}$ for efficiency. For ACPR, linearity (for a given $\Gamma_{L}$ ) decreases as $P_{i n}$ is increased. As such, it is expected that the limiting constant-ACPR surface will narrow as the Smith Tube is ascended, with fewer values of $\Gamma_{L}$ providing acceptable ACPR as $P_{i n}$ is increased. These trends are observed in the simulations and measurement examples shown.

\section{SimULATION RESUltS}

Load-pull simulations were performed over input power levels from $0 \mathrm{dBm}$ to $37 \mathrm{dBm}$ for a nonlinear FET model in the Keysight Technologies Advanced Design System (ADS) simulator. A constraint of ACPR $\leq-40 \mathrm{dBc}$ was imposed on the design. The maximum PAE providing ACPR $\leq-40 \mathrm{dBc}$ was found to be $36.79 \%$ at $P_{i n}=21 \mathrm{dBm}$ with $\Gamma_{L}=$ $0.800 / 180^{\circ}$. Traditionally measured load-pull contours are shown in Figures 2, 3, and 4 for load-pull measurements at input power values $P_{i n}=20,21$, and $22 \mathrm{dBm}$, respectively. The acceptable-ACPR region is shaded in each figure (the region of $\Gamma_{L}$ values providing ACPR $\leq-40 \mathrm{dBc}$ ). As $P_{i n}$ is increased, the acceptable region grows smaller. No acceptable region exists for $P_{\text {in }}=23 \mathrm{dBm}$.

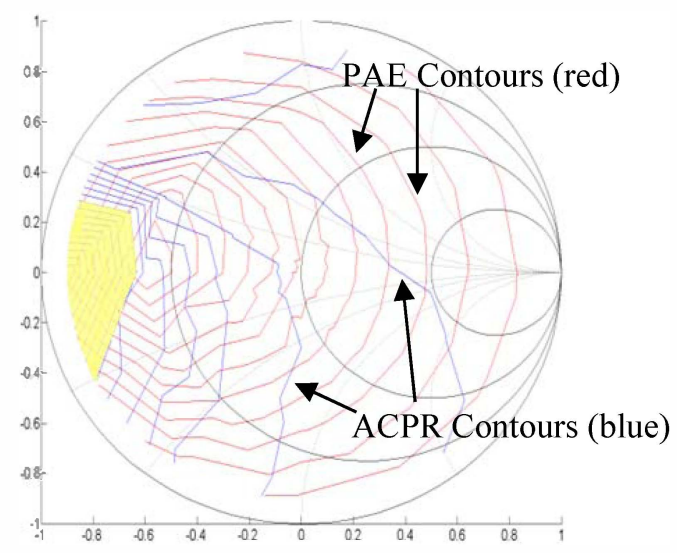

Fig. 2. Simulated PAE and ACPR load-pull contours for $P_{\text {in }}=20$ $\mathrm{dBm}$. The acceptable ACPR region (ACPR $\leq-40 \mathrm{dBc}$ ) is shaded.

Because the Smith Tube uses $P_{\text {in }}$ as the vertical axis, it allows display of the input-power-dependent load-pull data in one picture for design optimization. Figure 5 shows the ACPR surface representing ACPR $=-40 \mathrm{dBc}$ in the Smith Tube, with the surface representing $\mathrm{PAE}=36.79 \%$, the constrained optimum PAE. It can be seen that the two surfaces touch at one point, at which the surfaces are collinear.
For two-dimensional load-pull optimizations, PAE and ACPR contours are collinear at ACPR-constrained optimum solutions for PAE [10]. This collinearity also exists between the level surfaces of PAE and ACPR in three dimensions at the ACPRconstrained PAE optimum, as shown in Fig. 5.

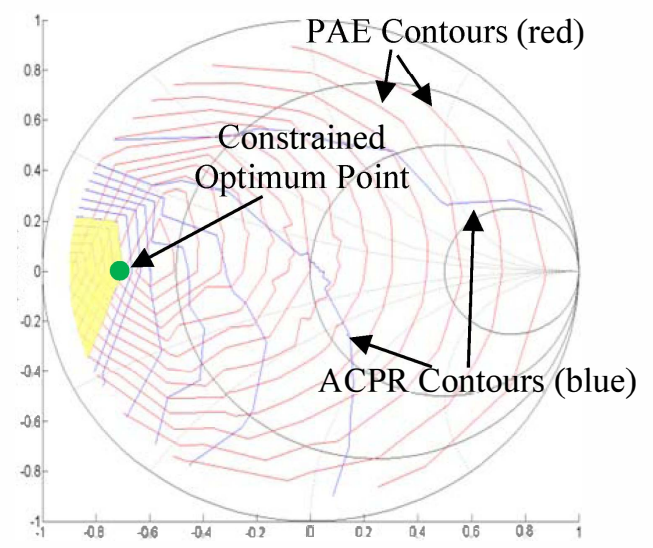

Fig. 3. Simulated PAE and ACPR load-pull contours for $P_{\text {in }}=21$ $\mathrm{dBm}$. The acceptable ACPR region (ACPR $\leq-40 \mathrm{dBc}$ ) is shaded. The ACPR-constrained optimum point for the design is indicated.

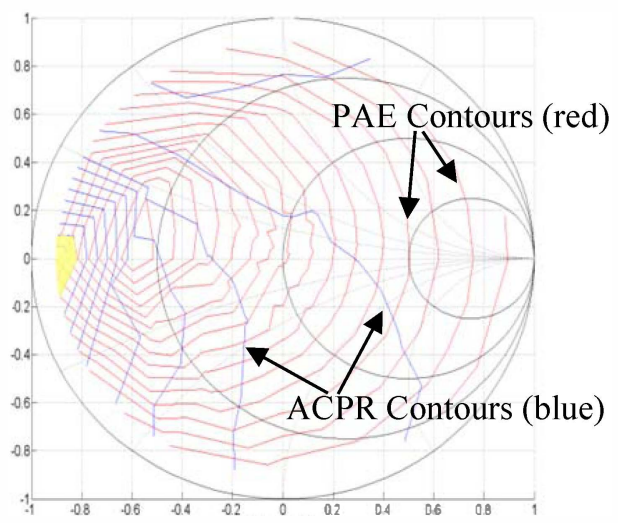

Fig. 4. Simulated PAE and ACPR load-pull contours for $P_{\text {in }}=22$ $\mathrm{dBm}$. The acceptable ACPR region $(\mathrm{ACPR} \leq-40 \mathrm{dBc})$ is shaded.

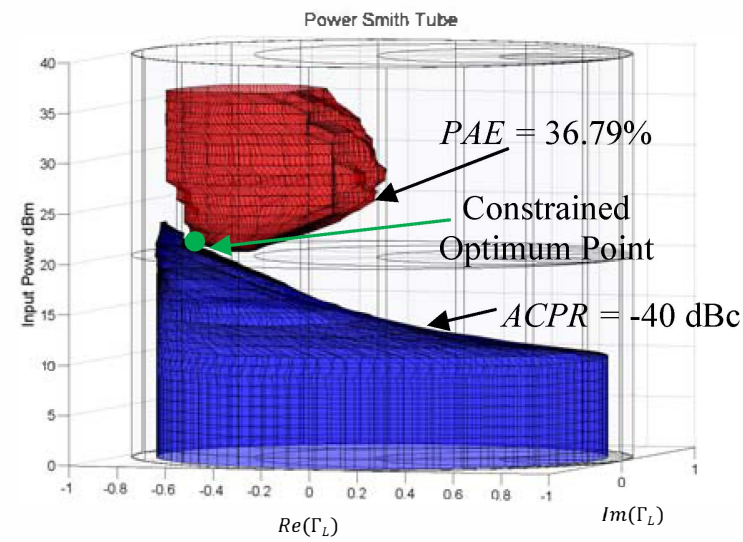

Fig. 5. Constant ACPR Surface from simulations for ACPR $=-40$ $\mathrm{dBc}$ and PAE surface for the maximum PAE from simulated loadpull data. The ACPR-constrained optimum solution occurs where the two surfaces intersect; the surfaces are collinear at this point. 


\section{MEASUREMENT RESUlts}

Measurement-based design was performed from multiplepower load-pull measurements on a Skyworks packaged amplifier (a different device is used for measurements to demonstrate the utility of the approach). A constraint of ACPR $\leq-27.5 \mathrm{dBc}$ was imposed. Figures 6,7 , and 8 show the load-pull results for $P_{\text {in }}=0,1$, and $2 \mathrm{dBm}$, respectively. The region of points providing $\mathrm{ACPR} \leq-27.5 \mathrm{dBc}$ is shown in each figure (Figure 7 shows the constrained optimum). The load-pull at $P_{i n}=2 \mathrm{dBm}$ provides an acceptable-ACPR region, but PAE results are lower than the maximum PAE found for $P_{\text {in }}=1 \mathrm{dBm}$.

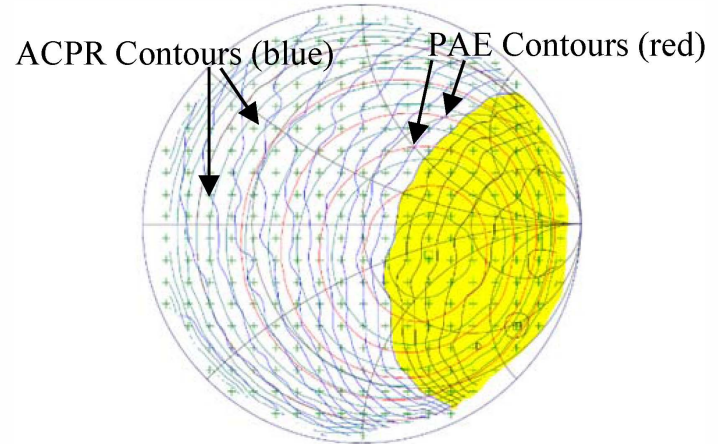

Fig. 6. Measured PAE and ACPR load-pull contours for $P_{\text {in }}=0$ $\mathrm{dBm}$. The acceptable ACPR region (ACPR $\leq-27.5 \mathrm{dBc}$ ) is shaded.

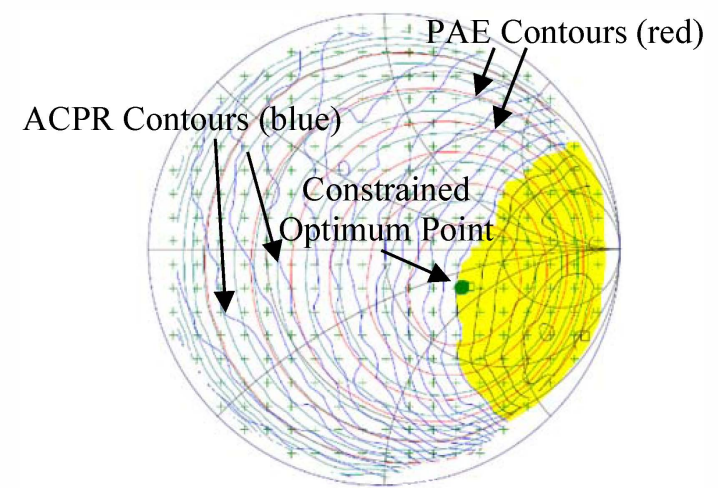

Fig. 7. Measured PAE and ACPR load-pull contours for $P_{\text {in }}=1$ $\mathrm{dBm}$. The acceptable ACPR region (ACPR $\leq-27.5 \mathrm{dBc}$ ) is shaded. The optimum point is indicated (this is the combination of $\Gamma_{L}$ and $P_{i n}$ providing the highest PAE while meeting ACPR requirements).

Figure 9 shows the three-dimensional visualization of the design in the Power Smith Tube. The limiting ACPR surface (ACPR $=-27.5 \mathrm{dBc})$ is plotted. The maximum PAE value within this surface is 6.707 percent, obtained with $\Gamma_{L}=0.35 /-28.4^{\circ}$ and $P_{i n}=1 \mathrm{dBm}$. Load-pull measurements should be performed at high enough power values to completely isolate the edge of the ACPR surface defining the ACPR-acceptable region. However, it is not recommended that the entire Smith Chart be measured (all values of $\Gamma_{L}$ ) at all power levels; the device could be harmed by measuring lowefficiency values of $\Gamma_{L}$ at high input power.

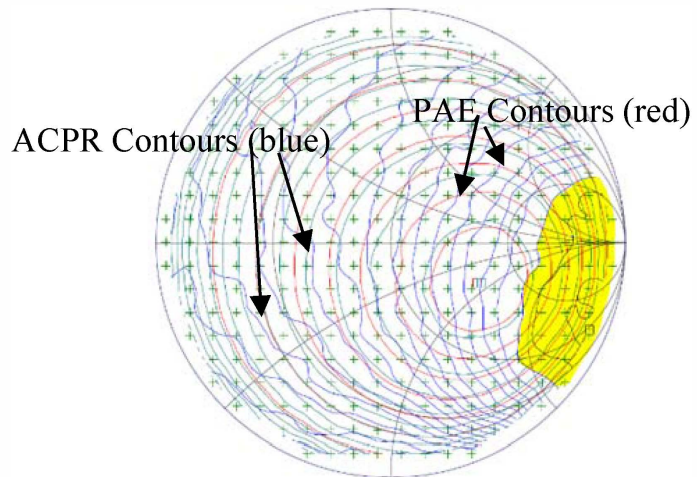

Fig. 8. Simulated PAE and ACPR load-pull contours for $P_{\text {in }}=2$ $\mathrm{dBm}$. The acceptable ACPR region $(\mathrm{ACPR} \leq-27.5 \mathrm{dBc})$ is shaded.

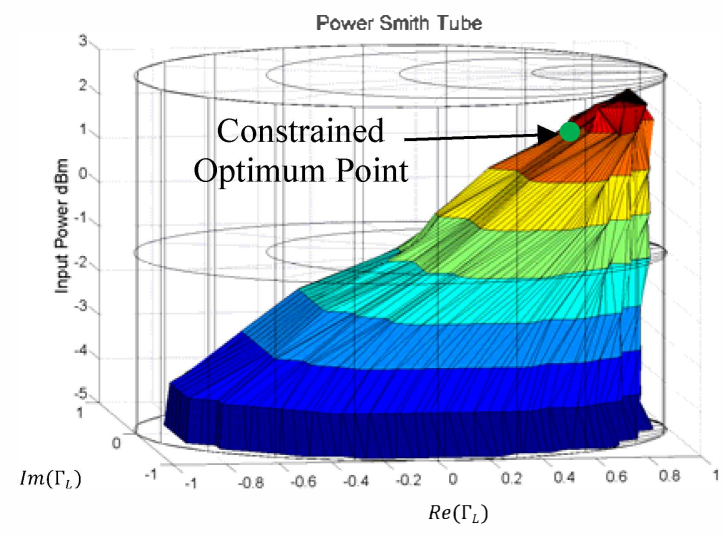

Fig. 9. Constant ACPR Surface from measured data for ACPR $=-27.5 \mathrm{dBc}$ from measured load-pull data. The constrained optimum solution occurs at the combination of $\Gamma_{L}$ and $P_{\text {in }}$ in this set producing the largest value of PAE.

\section{CONCLUSIONS}

The Power Smith Tube has been demonstrated as a useful visualization tool. It simplifies design for the linearityefficiency tradeoff. Joint design of the input reflection coefficient and input power to maximize PAE while keeping the ACPR within specified limitations has been demonstrated in this paper. Simulation and measurement results show that a surface of constant ACPR at the constraint value can be obtained, and the combination of load reflection coefficient and input power within this surface providing the largest PAE is chosen as the optimum. Designs for different devices in simulation and measurement have been demonstrated.

\section{ACKNOWLEDGMENTS}

The authors would like to thank Lawrence Cohen of the U.S. Naval Research Laboratory for valuable collaboration. This work has been funded by a grant from the National Science Foundation (Award Number ECCS-1343316). The authors would like to thank Keysight Technologies for cost-free loan of the Advanced Design System software. 


\section{REFERENCES}

[1] M. Fellows, M. Flachsbart, J. Barlow, C. Baylis, L. Cohen, and R.J. Marks II, "The Smith Tube: Selection of Radar Chirp Waveform Bandwidth and Power Amplifier Load Impedance Using Multiple-Bandwidth Load-Pull Measurements," 2014 IEEE Wireless and Microwave Technology Conference (WAMICON 2014), Tampa, Florida, June 2014.

[2] D.M. Snider, "A Theoretical Analysis and Experimental Confirmation of the Optimally Loaded and Overdriven RF Power Amplifier," IEEE Transactions on Electron Devices, Vol. ED-14, No. 12, December 1967, pp. 851-857.

[3] H.M. Nemati, C. Fager, U. Gustavson, R. Jos, and H. Zirath, "Design of Varactor-Based Tunable Matching Networks for Dynamic Load Modulation of High Power Amplifiers," IEEE Transactions on Microwave Theory and Techniques, Vol. 57, No. 5, May 2009, pp. 1110-1118.

[4] J..-S. Fu and A. Mortazawi, "Improving Power Amplifier Efficiency and Linearity Using a Dynamically Controlled Tunable Matching Network," IEEE Transactions on Microwave Theory and Techniques, Vol. 56, No. 12, December 2008, pp. 3239-3244.

[5] R. Hajji, F. Beauregard, and F.M. Ghannouchi, "Multitone Power and Intermodulation Load-Pull Characterization of Microwave Transistors Suitable for Linear SSPA's Design," IEEE Transactions on Microwave Theory and Techniques, Vol. 45, No. 7, July 1997, pp. 1093-1099.

[6] C. Zelley, "A Spherical Representation of the Smith Chart," IEEE Microwave Magazine, Vol. 8, No. 3, June 2007, pp. 60-66.

[7] Y. Wu, Y. Zhang, Y. Liu, and H. Huang, "Theory of the Spherical Generalized Smith Chart," Microwave and Optical Technology Letters, Vol. 51, No. 1, November 2008, pp. 95-97.

[8] A. Shamin, A.G. Radwan, and K.N. Salama, "Fractional Smith Chart Theory," IEEE Microwave and Wireless Components Letters, Vol. 21, No. 3, March 2011, pp. 117-119.

[9] J. Kretzschmar and D. Schoonaert, "Smith Charts for Lossy Transmission Lines," Proceedings of the IEEE, Vol. 57, No. 9, September 1969, pp. 1658-1660.

[10] J. Martin, C. Baylis, L. Cohen, J. de Graaf, and R.J. Marks II, "A Peak-Search Algorithm for Load-Pull Optimization of Power-Added Efficiency and Adjacent-Channel Power Ratio," IEEE Transactions on Microwave Theory and Techniques, Vol. 62, No. 8, August 2014, pp. 1772-1783. 